\title{
Mobile Ad-Hoc Networks: An Overview
}

\author{
Md. Sajid Anwer \\ Department of Computer Science, AISECT \\ University, Bhopal, India
}

\author{
Syed Hamid Hasan \\ Department of Information Systems, King \\ Abdulaziz University, Jeddah, Saudi Arabia.
}

\begin{abstract}
When more than 1 terminal/node/device, that has networking and communication capabilities, communicate with a similar device in the absence of a centralized admin or a situation where dynamic network of wireless nodes communicates amongst each other without any fixed infrastructure it is called MANET (Mobile Ad-Hoc Networks). MANET is autonomous wireless mobile host systems where the node are dynamically linked to each other, at times acting as routers as well. In this paper we will discuss a typical wired network, its characteristics, and its ever changing network configuration without limit of size or direction. Thus arriving at a need for a new routing protocol (optional path agreements) that will specify the nodes for this purpose. The ideal routing protocol must not only enable the identification of the best path but also enable the Ad Hoc Network to adapt to this route dynamically. We will also discuss types of MANET, its history along with approaches of wireless mobile. We will also touch upon the various routing protocol suggested for the Ad-Hoc Network along with possible applications of MANET, pros \& Cons, characteristic analysis and routing protocols.
\end{abstract}

\section{Keywords}

Ad Hoc Application, QoS, Mobile AdHoc sensor networks, Wireless mobile approach, AdHoc Routing protocol MANET, WSN, WMN.

\section{INTRODUCTION}

The development of wireless communications and computers has led to mobile computing becoming the center of computer communication on a very large scale. MANET (Mobile Ad Hoc Networks) are dynamic networks of wireless node that are primarily constructed by actions in the network, it is typically dynamic with limited features and bandwidth. The members of the network may be on Digital Camera, MP3 Player, Mobile Phone, PDA and Laptops etc. Mobility is the term used to describe action host that roam on different domain on the internet, they can even retain fixed IPs instead of obtaining new IP on each domain, also known as Mobile IP. IP addresses are managed primarily by the Mobile IP nodes using the by Home Agents while the Foreign Agents manage the routing and packet tunneling in the mobile nodes, this is same as a fixed network but, the Mobility provided AdHoc Networks are completely wireless. It can be based on network infrastructure that doesn't have a base station, every node can act as a router and be part of any link. MANET can be said to be the successor of the Military Radio network using mobile packets, with the advancement of technology ICs and equipment grow smaller in weight and size with lower power consumption. PCS (Personal Communications Systems) have evolved greatly in the form of mobile phones where information gathering, exchange and communication can happen at any time. It has become a necessity of life today. In a Military field operation which is a dangerous environment, the basic requirement of a network infrastructure, a base station, may not be available; Hence for communication between different units and the armed forces must rely on MANET, since it does not have the limitations of network infrastructure. Relief and rescue operation carried out either at sea or mountains cannot be expected to have a complete infrastructure apart from being pressed for time. Since the Ad-Hoc Network can use any mobile device and is completely wireless it is best suited for emergency relief and military operations. Today PCSs are really powerful and can be used for exchange even large amounts of data, such as big files, or even used for display applications. If we can link the devices in a temporary network, then we do not require expensive projection equipment. Bluetooth has attracted a lot of attention amongst the other WLAN technologies. If added to the MANET the Bluetooth would be able to enable connection between devices.

\section{BACKGROUND}

Communication and information technology are predominantly based on wireless, the traditional cellular and mobile network are limited by the need of support infrastructure like routers and base stations etc. MANET however eliminated the limitation of the traditional wireless networks. The Ad-Hoc networks are composed of more than two devices in a network with the absence of a centralized network controller, which latest in the evolution of mobile networks. Even though the primary consumer of MANET is still tactical military communication, but other applications are also considering the use of Ad-Hoc networks for its mobile networks. Possible uses are emergency services, rescue teams in natural disaster, law enforcement agencies, and sensor networks, however the application is not limited to such critical functions and the Ad-Hoc network can be used in al major segments of day to day communications.

PRNET [Packet Radio Networking] and DARPA[Defense Advance Research Project Agency] can be considered to the originators of Ad-Hoc Networks from as early as 1972 (1, 2, 3 ) the same lead to the evolution SURAN (Survivable adaptive radio networks) (4). It was initially instigated by the possible use of bandwidth sharing, and forward/store routing among other packet switching technologies in the MANET environment. <Done> Back then Routers and repeaters in the Packet Radio Network devices were mobile even though mobility was a very small part of the solutions so much so that advance protocols were considered great in 1970s. With advancements in micro-electronical technologies the entire network devices and nodes were integrated in Ad-Hoc Nodes. With the evolution of resilience, flexibility along with independence from fixed infrastructure and mobility made it an interesting option for use in the military technology. A crucial role has been played by Ad-hoc network in military communication technology and its associated research, e.g. NTDR [Near Term Digital Radio] or GloMo [Global Mobile informational system] programs $(5,6)$. Un-organized environments like search \& rescue, police and commercial companies have been utilizing Ad-Hoc networks apart from 
the military communication technology utilizing it. In 1990s commercial radio and wireless technologies become aware of the tremendous potential of MANET outside the domain of defense communication and technology.

\section{MANET TYPES}

\subsection{VANETs (Vehicular Ad-hoc Networks)}

VANET technology turns moving cars into the nodes of a network for creating mobile networks. VANET utilizes every participating car in the radius of 100-300 miters or each other by turning it into a node or wireless router, which connects with each other making a wide ranged network. Cars coming into the network join automatically while the ones going out of range move out of the network creating an Ad-hoc mobile internet. Fire and Police vehicles can communicate with each other using this technology as one of the primary users to integrate it into daily use.

\section{2 iMANET (Internet Based MANET)}

iMANET links Internet gateway node that are fixed with mobile nodes into an Ad-Hoc network. Standard Ad-Hoc routing algorithm is not applied directly in such kind of network. We can classify wireless network into 3 categories, MANET, Wireless networks and Fixed wireless networks. The basis of MANET is to establish a network without the assistance of a normal centrally controlled administrator found in a stand network infrastructure. These networks are essentially suitable for environments where there is no preexisting fixed network infrastructure or where it is not possible to establish such a fixed infrastructure.

\subsection{InVANETs (Intelligent VANET)}

InVANET defines using of VANET intelligently. Multiple AdHoc network technology such as ZigBee, IRA, Bluetooth, WiMax-IEEE 802.16, WAVE-IEEE 1609 and WiFi-IEEE 802.11 are integrated by InVANET for simple, effective, accurate and easy communications between vehicles moving in and out of the network dynamically. This technology can be utilized for media communication between participating vehicles or for even tracking of vehicles. InVANET can be utilized for telematics, infotainment, streaming communications and to define vehicle safety measures. VANET implements and utilizes a number of wireless technology like, WiMAX, Satellite, Cellular and DSRC (Dedicated Short Ranged Communication - a variant of $\mathrm{WiFi}$. VANET can be considered as an integral component of ITS (Intelligent Transportation System)

\section{ROUTING PROTOCOL}

To design communication \& networking protocol for MANET is a challenging task, since MANET is dynamic is nature. Designing of routing protocols is one of the most crucial part of communication process, since they help maintain and establish the multi-hop routes that enable data transfer/communication amongst the nodes. There has be extensive research carried out in the field of designing protocols that enable multi-hop routing. Some of these are DSDV, DSR, AODV and TORA $(6,7,8,9)$. Some of these protocols may be suitable in managing the communication and routing certain set of MANET application, however, most of them do not provide adequate support for more audio/video or multimedia intensive application. These kind of application necessitates QoS (Quality of Service) guarantees from the network. There has been work done in the field of providing QoS assurance for MANET applications and there have been various suggestions on which routing protocols would be able to provide such guarantees. Some protocols are able to assure QoS for link availability over specified paths primarily because routing protocol services are improved with better link availability predictions.

\subsection{Strategy for Routing Protocols}

We have 3 major strategies for Adhoc routing. The first is a proactive strategy and is referred to as Table-driven strategy, second is reactive strategy referred to as demand-driven or source-initiated. The third and the final strategy combines aspects and functionality of both the Table driven and the Demand driven strategies in order to give rise or a Hybrid strategy.

4.1.1 Table Driven: In in routing approach each and every nodes has information about the complete network routing paths and its continuously updated. Whenever a packet needs to be forwarded the required route is already available within the node, hence there is no delay/wait time for obtaining the best possible route for information relay. But, since the topology of the network is very dynamic and there are nodes being added and taken off on the go, hence the network must spend a major portion of the available resources in keeping the network information upto date. Optimized Link-States routing \& DSDV(Destination Sequenced Distances Vectors)(6) are example of this kind of routing strategy.

4.1.2 Demand Driven: Here only active destination routes are maintained by the nodes. Whenever a new destination is required a route search must happen. This provides a saving in terms of communication overhead in maintaining all routes, however there must be a delay in setting up new routes via route search. This kind of setup is best suited for Ad-hoc environment since it saves battery power by restricting sending and receiving of communication in the form of advertising.

4.1.3 Hybrid strategies: Here the entire network is divided into clusters or zones, the nodes within the zone are controlled/governed by proactive strategy while the nodes outside each zone follow the reactive strategy when communication is to happen between nodes of different clusters. Large networks that is usually divided into partitions or clusters are most apt for applying the hybrid strategy, since it strikes a balance between resource conservation and network performance.

\subsection{DSDV (Destination Sequence Distance Vectors)}

Amongst the various routing protocols applied to MANET the primary is DSDV (Destination Sequence Distance Vectors), this protocol is commonly referred as the Bellman Ford's Distance Vector adaptation for MANET. Each node inside the network stores the routing table and these are used for transmitting packets between nodes. The table lists all possible destination nodes, next hop nodes and total number of hops required to reach the destination node. Every entry in the routing table is sequenced by a number that is provided from the destination nodes. Freshness of the route indication and loop avoidance is achieved by the sequence number in AODV. Routing table consistency is maintained in this rapidly changing and dynamic environment by periodic 
broadcast of information by the nodes, along with broadcasts that are followed by major information updates/changes. Hence, DSDV falls under the umbrella of proactive protocol. Multicasts or broadcasts are used for advertising route information. Reduction of information published by each advertisement is done by defining two kinds of packets. First one named the "full dump" contains all available information related to routing. Second one the "incremental" contains the information that has changed from the time the last "full dump" was sent out. Frequency of Full dumps reduce considerably, if there is no change in the mobile hosts/ nodes. However, if the movement of nodes become frequent and incremental packets reach the size of NPDU (Network Protocols Data Units) a full dump packet is then scheduled for transmission. Traffic is further reduced by delaying advertisements pertinent to routes that are still unstable. Any new information about routing, received by a mobile host is compared with the existing information that is received via previous packet transmissions. The routes that have the most updated sequence are used thereon and routes with old/obsolete sequence number are cast aside. In case of two routes having the same sequence number, the choice is made on the basis of other factors like number of hops in the route etc. In case the link in the route for next hops are broken, the route connecting the next hop is instantly allocated the latest sequence number with infinite metric. All these changes are instantaneously transmitted throughout the network in the form of an information packet for routing.

\section{CONCLUSION}

Through this paper we discussed Ad-Hoc network, various types of MANETs and the routing protocol strategies and their advantages. Various kinds of MANET explained the concept of dynamic/moving nodes inside the network in detail. This study provides a basic understanding of the AdHoc networks and the numerous applications that these networks can be applied to. The future of this study is the further research required in the field of improving the accuracy and effectiveness of communication inside the MANET networks.

\section{REFERENCES}

[1] Toh. C.K., 2002. Ad Hoc Mobile Wireless Networks Protocols and Systems. Prentice Hall,Inc

[2] Freebersyser, J. A., and Leiner, B. A DoD perspective on mobile ad hoc networks. In: Perkins, C. (Ed.) Ad Hoc Networking, Addison Wesley, Reading, MA, 2001, pp. $29-51$.

[3] J. Jubin and J.D. Tornow, "The DARPA Packet Radio Network Protocols", proceedings of the IEEE, vol. 75, no. 1, January 1987, pp.21-32.

[4] J. A. Freebersyser and B. Leinerr, "A DoD perspective on mobile ad hoc networks," in Ad Hoc Networking, C. E. Perkin, Ed. Addison-Wesley, 2001, pp. 29-51.

[5] R. Ruppe, S. Griswald, P.Walsh, and R. Martin, "Near term digital radio (NTDR) system", in Proceedings of IEEE MILCOM, vol. 3, November 1997, pp.1282 1287.

[6] B. Leiner, R. Ruth, and A. R. Sastry, "Goals and challenges of the DARPA GloMo program," IEEE
Personal Communications, vol. 3, no. 6, pp. 34-43, December 1996.

[7] Vani A and Rao D, "Providing of Secure Routing against Attacks in MANETs" International Journal of Computer Applications (0975 - 8887) Volume 24- No.8, June 2011.

[8] Senthilkumar P., Baskar M. and Saravanan K., "A Study on Mobile Ad-Hock Networks (MANETS)", JMS, Vol. No.1, Issue No.1, September 2011.

[9] Satria Mandala, Md. Asri Ngadi and A.Hanan Abdullah, "A Survey on MANET Intrusion Detection" IJCSS, Vol No 2, Issue 1, 2007.

[10] Ruchi R., Dawra M., "Performance characterization of AODV protocol in MANET", IJARCET, Vol No 1, Issue No 3, May2012.

[11] D.Sivaganesan1 and Dr.R.Venkatesan, "Performance Analysis of Broadcasting in Mobile ad hoc networks using cluster approach", IJASUC Vol No.1, Issue No.2, June 2010.

[12] Sreerama M and Venkat D., "Performance Evalution of MANET Routing Protocols using Reference Point Group Mobility and Random WayPoint Models", IJASUC Vol No.2, Issue No.1, March 2011.

[13] Murty S, Dastagiraiah C. and Kumar A, "Analysis of MANET routing Protocols Using Random waypoint Model in DSR", IJASUC Vol No .2, Issue No.4, December 2011.

[14] Chaudhary D., "Bee-Inspired Routing Protocols for mobile Ad HOC Network (MANET)", JETWI, VOL No. 2, Issue No. 2, MAY 2010.

[15] Koshti D and Kamoji S, "Comparative study of Techniques used for Detection of Selfish Nodes in Mobile Ad hoc Networks" IJSCE, Vol 1, Issue 4, $2011 \mathrm{~W}$ Lien and Feng Yi "A Threshold-Based Method for Selfish Nodes Detection in MANET", 978-1-4244-76404/10/2010 IEEE.

[16] W Lien and Feng Yi, "A Threshold-Based Method for Selfish Nodes Detection in MANET", IEEE, 2010.

[17] Sukumaran S, Venkatesh. J and Arunkorath, "A Survey of Methods to mitigate Selfishness in Mobile Ad hoc Networks" IJICT, Vol 1, Issue No. 2, June 2011.

[18] Rani et al., International Journal of Advanced Research in Computer Science and Software Engineering 3(3), March - 2013, pp. 135-138

[19] Roy B., Banik S., Dey P., Sanyal S and Chaki N, "Ant Colony based Routing for Mobile Ad-Hoc Networks towards Improved Quality of Services", JETCIS, Vol. No 3, Issue No. 1, January 2012.

[20] P.V.Jani, "Security within Ad-Hoc Networks," Position Paper, PAMPAS Workshop, Sept. 16/17 2002.

[21] K. Biswas and Md. Liaqat Ali, "Security threats in Mobile Ad-Hoc Network", Master Thesis, Blekinge Institute of Technology" Sweden, 22nd March 2007. 